\title{
Search for GeV-Scale Sterile Neutrinos Responsible for Active Neutrino Oscillations and Baryon Asymmetry of the Universe
}

\author{
S. N. Gninenko, ${ }^{1}$ D. S. Gorbunov, ${ }^{1,2}$ and M. E. Shaposhnikov ${ }^{3}$ \\ ${ }^{1}$ Institute for Nuclear Research of Russian Academy of Sciences, 117312 Moscow, Russia \\ ${ }^{2}$ Moscow Institute of Physics and Technology, 141700 Dolgoprudny, Russia \\ ${ }^{3}$ Institut de Théorie des Phénomènes Physiques, (École Polytechnique Fédérale de Lausanne), \\ 1015 Lausanne, Switzerland \\ Correspondence should be addressed to D. S. Gorbunov, gorby@ms2.inr.ac.ru
}

Received 27 July 2012; Accepted 25 September 2012

Academic Editor: Gian Luigi Fogli

Copyright (c) 2012 S. N. Gninenko et al. This is an open access article distributed under the Creative Commons Attribution License, which permits unrestricted use, distribution, and reproduction in any medium, provided the original work is properly cited.

\begin{abstract}
Standard Model fails to explain neutrino oscillations, dark matter, and baryon asymmetry of the Universe. All these problems can be solved with three sterile neutrinos added to SM. Quite remarkably, if sterile neutrino masses are well below the electroweak scale, this modificationNeutrino Minimal Standard Model (vMSM) — can be tested experimentally. We discuss a new experiment on search for decays of $\mathrm{GeV}$-scale sterile neutrinos, which are responsible for the matter-antimatter asymmetry generation and for the active neutrino masses. If lighter than $2 \mathrm{GeV}$, these particles can be produced in decays of charm mesons generated by high energy protons in a target, and subsequently decay into SM particles. To fully explore this sector of $v$ MSM, the new experiment requires data obtained with at least $10^{20}$ incident protons on target (achievable at CERN SPS in future) and a big volume detector constructed from a large amount of identical single modules, with a total sterile neutrino decay length of few kilometers. The preliminary feasibility study for the proposed experiment shows that it has sensitivity which may either lead to the discovery of new particles below the Fermi scale-right-handed partners of neutrinos-or rule out seesaw sterile neutrinos with masses below $2 \mathrm{GeV}$.
\end{abstract}

\section{Introduction}

The discovery of neutrino oscillations provides an undisputed signal that the Standard Model (SM) of elementary particles is not complete. However, what kind of new physics it brings to us remains still unclear: we do not know yet the properties of new particles which are believed to be behind this phenomenon. An attractive possibility is the extension of the SM by three right-handed neutrinos, making the leptonic sector similar to the quark one, see Figure 1. 

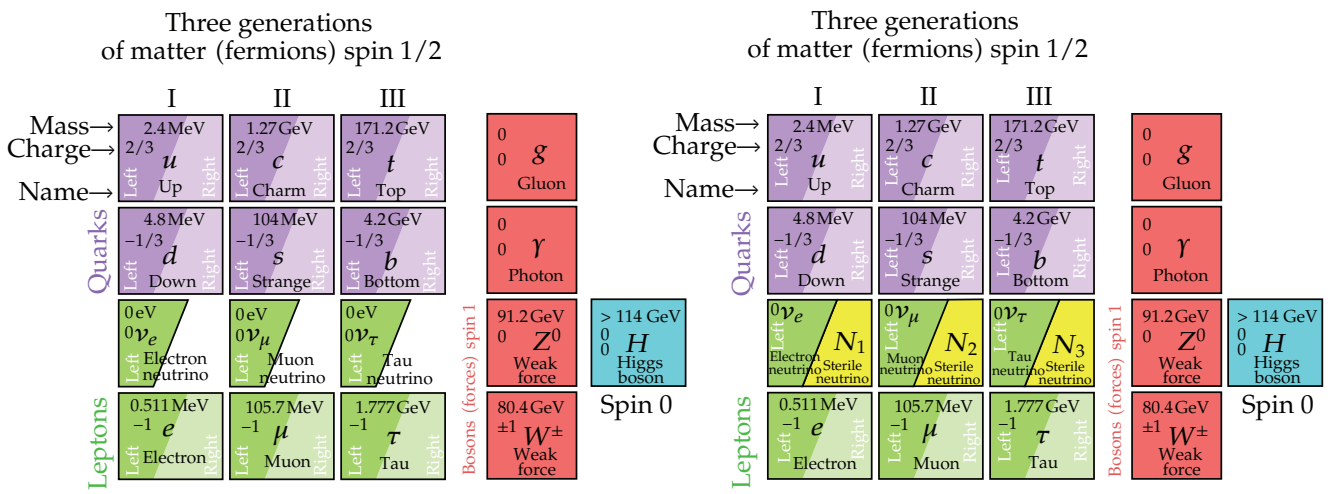

Figure 1: Particle content of the SM and its minimal extension in neutrino sector. In the SM (left) the righthanded partners of neutrinos are absent. In the vMSM (right) all fermions have both left and right-handed components.

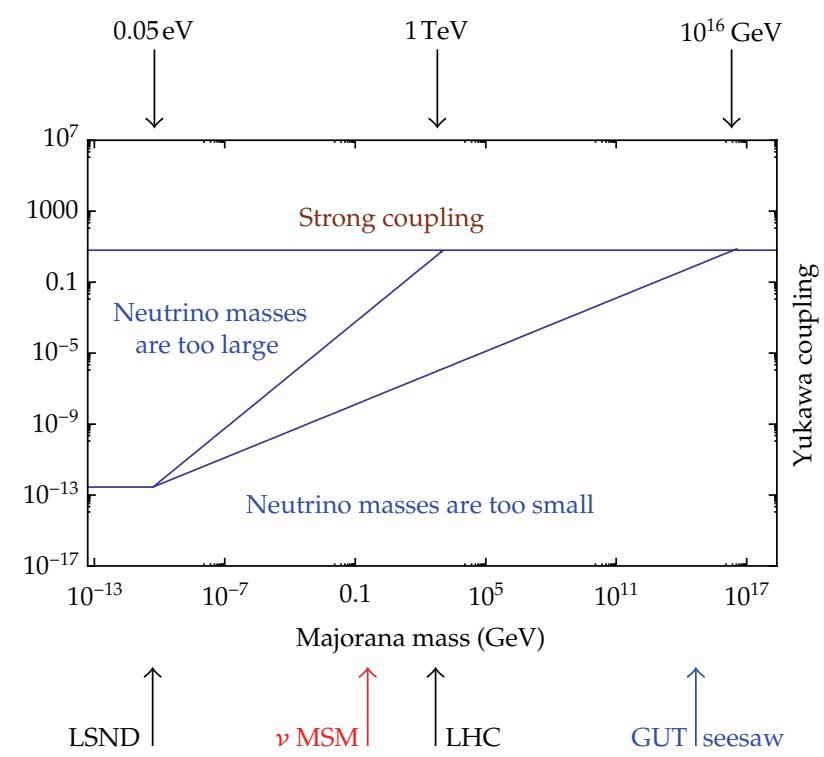

Figure 2: The admitted values of the Yukawa couplings of sterile neutrinos as a function of their seesaw Majorana masses.

The masses $M$ and Yukawa couplings $Y$ of new leptons remain largely unknown. Basically, $M$ can have any value between zero (corresponding to Dirac neutrinos) and $10^{16} \mathrm{GeV}$, whereas $Y$ can vary from $10^{-13}$ (Dirac neutrino case) to $\sim \pi$ (the onset of the strong coupling). The admitted region is sketched in Figure 2.

Independently on their mass, the new Majorana leptons can explain oscillations of active neutrinos. So, an extra input is needed to fix their mass range. It can be provided by the LHC experiments.

Suppose that the resonance found at the LHC by Atlas and CMS in the region 125$126 \mathrm{GeV}$ is indeed the Higgs boson of the Standard Model. This number is remarkably close to the lower limit on the Higgs mass coming from the requirement of the absolute stability of 
the electroweak vacuum and from Higgs inflation, and to prediction of the Higgs mass from asymptotic safety of the Standard Model (see detailed discussion in [1] and in a proposal submitted to European High Energy Strategy Group by Bezrukov et al. [2]). The existence of the Higgs boson with this particular mass tells that the Standard Model vacuum is stable or metastable with the life-time exceeding that of the Universe. The SM in this case is a valid effective field theory up to the Planck scale, and no new physics is required above the Fermi scale from this point of view. Suppose also that the LHC finds no new particle and no deviations from the Standard Model. In this case the "naturalness paradigm", leading the theoretical research over the last few decades will be much less attractive, as the proposals for new physics stabilizing the electroweak scale by existence of new particles in the $\mathrm{TeV}$ region and based on low energy supersymmetry, technicolor or large extra dimensions would require severe fine-tunings.

The solution of the hierarchy problem does not require in fact the presence of new particles or new physics above the Fermi scale. Moreover, the absence of new particles between the electroweak and Planck scales, supplemented by extra symmetries (such as the scale invariance) may itself be used as an instrument towards a solution of the problem of stability of the Higgs mass against radiative corrections (for detailed arguments see [3-5]).

Even regardless the hierarchy problem, it is clear that the Standard Model of elementary particles is not complete. It is in conflict with several observations. These are nonzero neutrino masses and oscillations discussed above, the excess of matter over antimatter in the Universe, and the presence of nonbaryonic dark matter. Any model of physics beyond the SM (BSM) should be able to deal with the experimental troubles of the SM.

Guided by the arguments steaming from alternative solutions to the hierarchy problem it is natural to ask whether the observational problems of the SM can be solved by new physics below the Fermi scale. And the answer is affirmative: an economic way to handle in a unified way the problems of neutrino masses, dark matter, and baryon asymmetry of the Universe is to add to the SM three Majorana singlet fermions with masses roughly of the order of masses of known quarks and leptons. This theory is called the $v$ MSM, for "Neutrino Minimal Standard Model" (for a review see [6]). The lightest of the three new leptons is expected to have a mass from $1 \mathrm{keV}$ to $50 \mathrm{keV}$ and plays the role of the dark matter particle (see detailed discussion in [6] and in a proposal submitted to European High Energy Strategy Group by Boyarsky et al. [7]). Two other neutral fermions are responsible for giving masses to ordinary neutrinos via the seesaw mechanism at the electroweak scale and to creation of the baryon asymmetry of the Universe. The masses of these particles and their couplings to ordinary leptons are constrained by particle physics experiments and cosmology. Two leptons should be almost degenerate, forming thus nearly Dirac fermion (this is coming from the requirement of successful baryogenesis). For comparison, we show in Figure 3 the summary of different possibilities for the masses of Majorana leptons.

A lot of experimental efforts were devoted to the direct search of Majorana neutral leptons in the past [8-16]. No new particles were found, but several constraints on their mixing angles with ordinary leptons were derived. The interest to these searches declined considerably at nineties, most probably due to the theoretical prejudice that the masses of Majorana leptons should be associated with the Grand Unified scale $\sim 10^{16} \mathrm{GeV}[17-20]$, making their direct search impossible. As a result, no dedicated searches of relatively light neutral leptons were performed in the last decade, with an exception of several experiments [13-15] related to the so-called Karmen anomaly [21]. Today, the reason for the negative result became clear: in the domain of parameters accessible to most of these experiments the neutral leptons 


\begin{tabular}{|c|c|c|c|c|c|c|c|c|}
\cline { 2 - 9 } \multicolumn{1}{c|}{} & $\begin{array}{c}N \\
\text { mass }\end{array}$ & $\begin{array}{c}v \\
\text { masses }\end{array}$ & $\begin{array}{c}\text { eV } v \\
\text { anoma- } \\
\text { lies }\end{array}$ & BAU & DM & $\begin{array}{c}M_{H} \\
\text { stability }\end{array}$ & $\begin{array}{c}\text { Direct } \\
\text { search }\end{array}$ & $\begin{array}{c}\text { experi- } \\
\text { ment }\end{array}$ \\
\hline $\begin{array}{c}\text { GUT } \\
\text { seesaw }\end{array}$ & $\begin{array}{c}10-16 \\
10 \mathrm{GeV}\end{array}$ & Yes & No & Yes & No & No & No & - \\
\hline EWSB & $\begin{array}{c}2-3 \\
10 \mathrm{GeV}\end{array}$ & Yes & No & Yes & No & Yes & Yes & LHC \\
\hline$v$ MSM & $\mathrm{keV-GeV}$ & Yes & No & Yes & Yes & Yes & Yes & $\begin{array}{c}\mathrm{a}^{\prime} \text { la } \\
\text { CHARM }\end{array}$ \\
\hline $\begin{array}{c}v \\
\text { scale }\end{array}$ & $\mathrm{eV}$ & Yes & Yes & No & No & Yes & Yes & $\begin{array}{c}\text { a'la } \\
\text { LSND }\end{array}$ \\
\hline
\end{tabular}

Figure 3: This table shows whether a given choice of the mass of sterile neutrinos can explain neutrino masses and oscillations, accommodate $\mathrm{eV}$ neutrino anomalies, lead to baryogenesis, provide a dark matter candidate, ensure the stability of the Higgs mass against radiative corrections, and is directly searched at some experiments.

interact too strongly to produce the baryon asymmetry of the Universe, not satisfying one of the Sakharov conditions for baryogenesis.

The recent theory developments provide a guideline for the required experimental sensitivity [22], which was absent at the time when the early experiments were made. The aim of the present work is to discuss the experimental signatures of the existence of these particles and estimate the parameters of the experiment of beam-target type required to fully explore the model parameter space if sterile neutrinos are lighter than $D$-mesons (see a proposal submitted to European High Energy Strategy Group by Gorbunov and Shaposhnikov [23]).

The paper is organized as follows. In Section 2 we give the essential features of the model and discuss the constraints on new particles. Section 3 is devoted to general description of experiments on searches for sterile neutrinos of masses in $\mathrm{GeV}$ range. In Section 4 we present an estimate of sensitivity required to test the model and suggest the experimental setup to accomplish the ultimate task. Section 5 contains conclusions.

\section{The Model and Constraints on Properties of New Particles}

The $v$ MSM is described by the most general renormalizable Lagrangian of all the SM particles and 3 singlet fermions. For the purpose of the present discussion we take away from it the lightest singlet fermion $N_{1}$ (the "dark matter sterile neutrino"), which is coupled extremely weakly to the ordinary leptons. In addition, we take $N_{2}$ and $N_{3}$ degenerate in mass, $M_{2}=M_{3}=M$. The approximate equality of the masses of $N_{2,3}$ comes from requirement of resonance production of the baryon asymmetry of the Universe. Then a convenient parameterization of the mass term and the interaction of $N^{\prime}$ s with the leptons of SM is given in terms of three parameters $(M, \epsilon, \eta)$ :

$$
L_{\text {singlet }}=\left(\frac{M \sum m_{i}}{2 v^{2}}\right)^{1 / 2}\left[\frac{1}{\sqrt{\epsilon e^{i \eta}}} \bar{L}_{2} N_{2}+\sqrt{\epsilon e^{i \eta}} \bar{L}_{3} N_{3}\right] \widetilde{H}-M \bar{N}_{2}^{c} N_{3}+\text { h.c. }
$$


where $L_{2}$ and $L_{3}$ are the combinations of the left-handed doublets $L_{e}, L_{\mu}$, and $L_{\tau}$,

$$
L_{2}=\sum_{\alpha} x_{\alpha} L_{\alpha}, \quad L_{3}=\sum_{\alpha} y_{\alpha} L_{\alpha}
$$

with $\sum_{\alpha}\left|x_{\alpha}\right|^{2}=\sum_{\alpha}\left|y_{\alpha}\right|^{2}=1$. In (2.1) $v=246 \mathrm{GeV}$ is the vacuum expectation value of the Higgs field $H, \widetilde{H}_{i}=\epsilon_{i j} H_{j}^{*}$, and $m_{i}$ are the active neutrino masses. Note that one of them is negligibly small in comparison with others in the $v$ MSM [6], so that $\sum m_{i}=\kappa m_{\text {atm }}$ with $m_{\mathrm{atm}} \equiv \sqrt{\left|\Delta m_{\mathrm{atm}}^{2}\right|} \simeq 0.05 \mathrm{eV}$ being the atmospheric neutrino mass difference, $\mathcal{\kappa}=1$ (2) for normal (inverted) hierarchy of neutrino masses. Mixing parameters $x_{\alpha}$ and $y_{\alpha}$ can be expressed through the parameters of the active neutrino mixing matrix (explicit relations can be found in [24]). The parameter $\epsilon$ (by definition, $\epsilon<1$ ) and the CP-breaking phase $\eta$ cannot be determined by neutrino oscillation experiments.

If the value of mass parameter $M$ is fixed, smaller $\epsilon$ yields stronger interactions of singlet fermions to the SM leptons. This leads to equilibration of these particles in the early Universe above the electroweak temperatures, and, therefore, to erasing of the baryon asymmetry. In other words, the mixing angle $U^{2}$ between neutral leptons and active neutrinos must be small, explaining why these new particles have not been seen previously. For small $\epsilon$,

$$
U^{2}=\frac{\sum m_{i}}{4 M \epsilon}
$$

The region, where baryogenesis is possible in $\left(U^{2}, M\right)$ plane is shown in Figure 4 . We also plot there the exclusion regions coming from different experiments such as CHARM [10], NuTeV [13], and CERN PS191 experiment [11, 12] (see also discussion of different experiments in $[25,26])$. Only CERN PS191 significantly entered into the cosmologically interesting part of the parameter space of the $v$ MSM for sterile neutrinos lighter than kaon. In Figure 5 we present the expected lifetime of the singlet fermions in an experimentally interesting region $M<1.8 \mathrm{GeV}$.

As we see, the mass of these particles is limited from below by current experiments and cosmological considerations by $\sim 100 \mathrm{MeV}$, while no known solid upper bound, better than the electroweak scale, can be applied. At the same time, various considerations indicate that their mass may be in $\mathcal{O}(1) \mathrm{GeV}$ region [24].

\section{General Considerations for the Experiment}

The most efficient mechanism of sterile neutrino production is associated with weak decays of heavy mesons and baryons, see left panel of Figure 6 for examples of relevant two- and three-body decays. Therefore, from experimental point of view, the distinct mass ranges are associated with the masses of mesons in which sterile neutrinos can be created: below $500 \mathrm{MeV}$ ( $K$-meson), between $500 \mathrm{MeV}$ and $2 \mathrm{GeV}$ ( $D$-mesons), between 2 and $5 \mathrm{GeV}(B$ mesons), and above $5 \mathrm{GeV}$. We will concentrate here on the masses below the charm threshold, the higher values will be discussed shortly in Section 5.

The mechanism works as follows. Heavy mesons (and baryons) can be produced by energetic protons scattering off the target material. As a reference machine we take CERN 
6

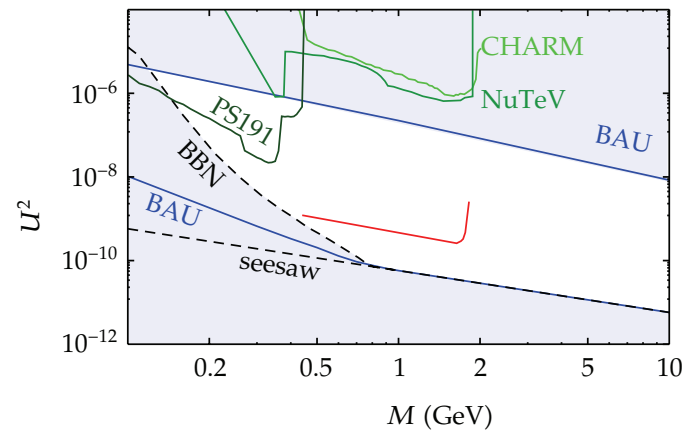

(a)

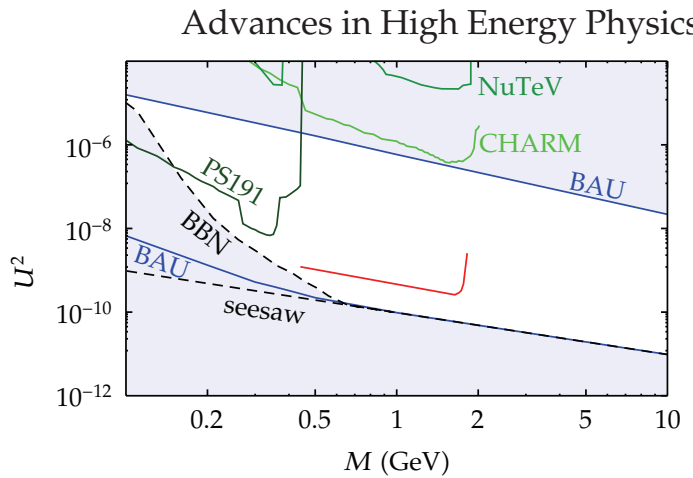

(b)

Figure 4: The allowed region of parameters of sterile neutrinos, responsible for neutrino oscillations (region above the dotted "seesaw" line) and for baryo/leptogenesis (region between two black solid lines). Sterile neutrinos with the parameters in the shaded region to the left of the "BBN" line would spoil predictions of primordial nucleosynthesis. Accelerator experiments, searching for heavy neutral leptons exclude regions above green lines. (a) restrictions for normal hierarchy (b) inverted hierarchy. Adopted from [27]. The region above the red curve can be probed with a single section of the detector of length $l_{\|} \sim 100 \mathrm{~m}$, height $5 \mathrm{~m}$ and width $l_{\perp} \sim 5 \mathrm{~m}$, placed at a distance of about hundred meters, see the text for details.

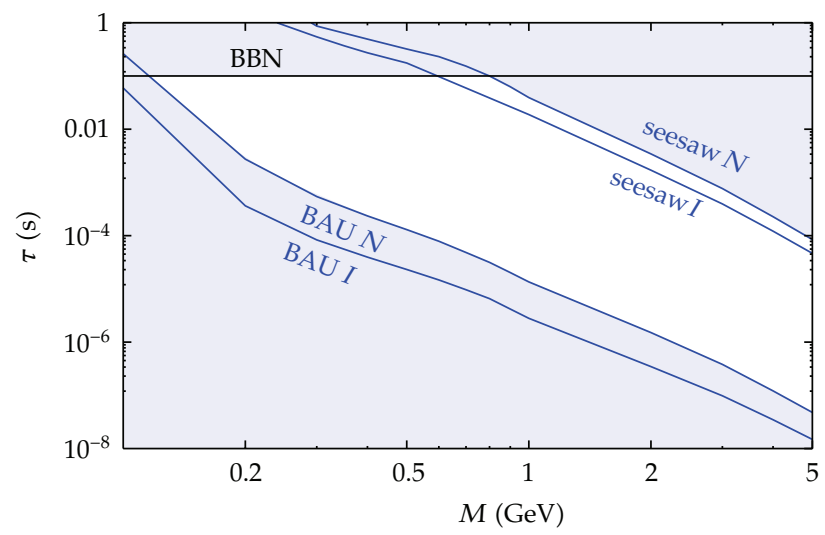

Figure 5: Constraints on the sterile neutrino lifetime $\tau_{N}$ coming from the baryon asymmetry of the Universe (blue solid lines: "BAU $N$ " refers to the normal hierarchy, "BAU I" refers to the inverted hierarchy), from the seesaw mechanism (blue solid lines: "Seesaw $N$ " refers to normal hierarchy, "Seesaw $I^{\prime \prime}$ refers to the inverted hierarchy) and big bang nucleosynthesis (black line: "BBN"). The allowed region of parameter space is in white. Limits from direct searches presented in Figure 4 are not outlined.

SPS with beam energy of $400 \mathrm{GeV}$. Here the relevant heavy hadrons contain charm as the heaviest quark: $D, D_{s}, \Lambda_{c}, D^{*}, \ldots$ With typical weak lifetime (in the rest frame) of about $10^{-10} \mathrm{~s}$ these mesons mostly decay before further interaction in the target, no matter how thick it is. Sterile-active neutrino mixing gives rise to sterile neutrino production in weak decays of the heavy mesons (note that searches for those heavy hadron decays is one of the possible strategy of exploring the $v$ MSM parameter space in full, which at the present level of experimental technique seems unrealistic, see discussion in Section 5). For charmed mesons typical branching ratios expected for the $v$ MSM parameter space are at the level of [22]

$$
\operatorname{Br}(D \longrightarrow N) \sim 10^{-8}-10^{-12},
$$

(referring to upper and lower limits in Figure 4, resp.). 

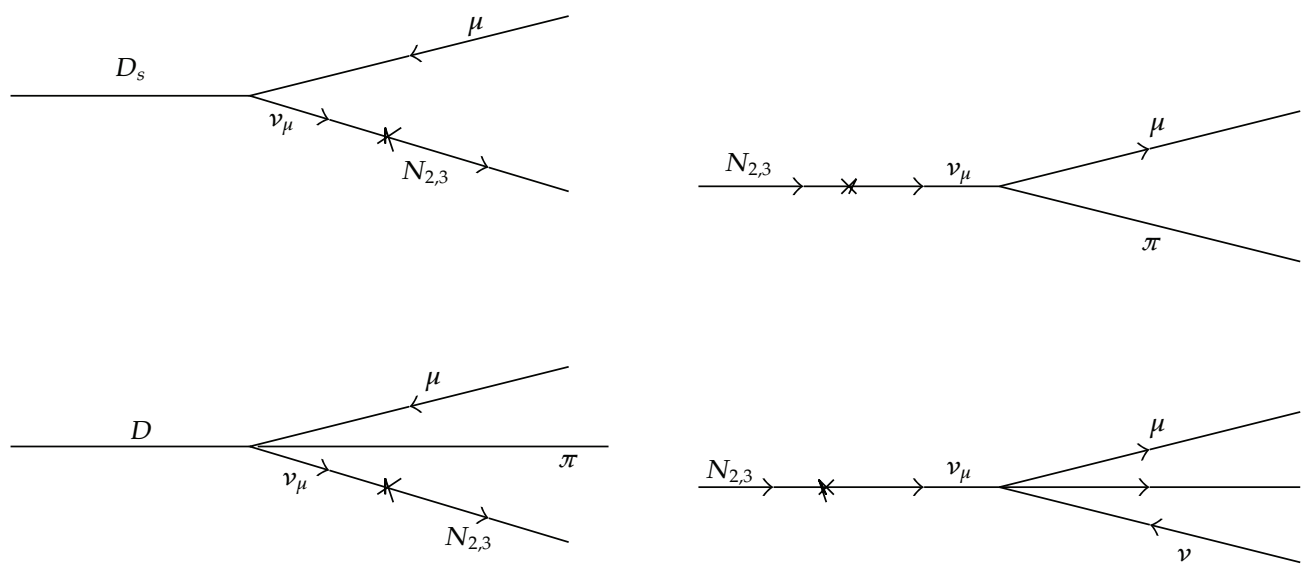

Figure 6: Left panel: Feynman diagrams of meson decays to heavy sterile neutrinos. Right panel: Feynman diagrams of sterile neutrino decays.

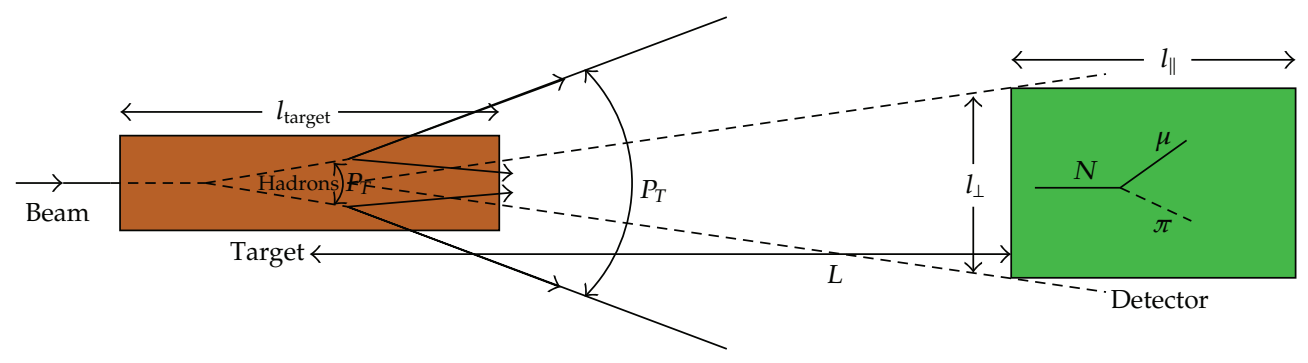

Figure 7: Sketch of typical beam-target experiment on searches for sterile neutrino decays.

These sterile neutrinos further weakly decay to the SM particles due to mixing with active neutrinos. In the $v$ MSM mixing angles are small, see Figure 4 , hence the sterile neutrinos live much longer (see Figure 5) as compared to that of the weakly decaying SM particles of similar masses. Before the decay (and with account of $\gamma$-factor) relativistic sterile neutrinos would cover quite a large distance significantly exceeding ten kilometers. Hence, sterile neutrino decays into SM particles due to mixing with active neutrino can be searched for in the near detector, see Figure 7. Two examples of the interesting decay modes are presented in right panel of Figure 6. More examples and typical patterns of sterile neutrino decay branching ratios expected within the $v$ MSM can be found in [22].

Let us first make rough estimates of length-scale of the detector needed to fully explore the $v$ MSM parameter space for sterile neutrino masses in the interesting range $M_{N} \sim 0.5-$ $2 \mathrm{GeV}$, where sterile neutrinos are dominantly produced in charmed hadron decays. Let $P_{T}$ and $P_{L}$ be average transverse and longitudinal momenta of produced sterile neutrinos. We start with detector of a special geometry designed to cover entirely the solid angle $\sim \pi P_{T}^{2} / P_{L}^{2}$ around the beam axis. Then all the sterile neutrinos travel through the fiducial volume of the detector which has a conical form. For $400 \mathrm{GeV}$ proton beam operating at SPS the charm 


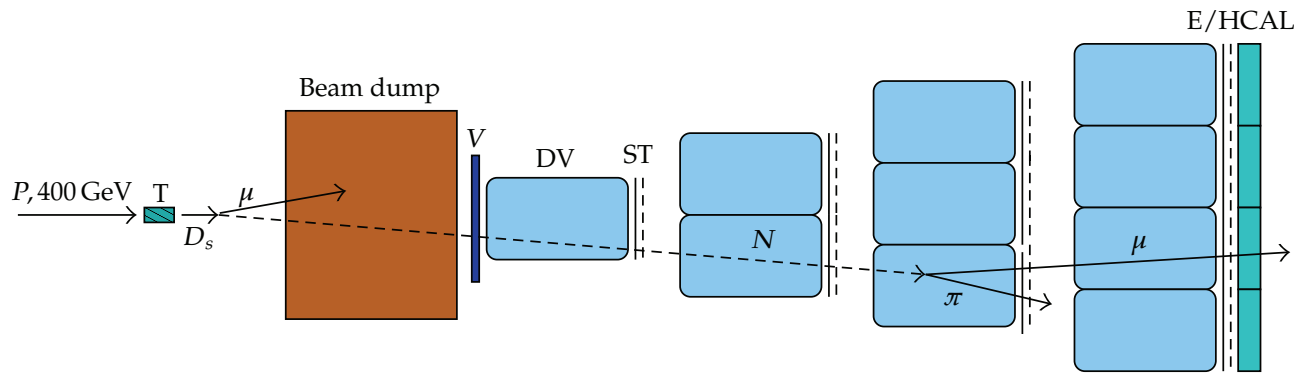

Figure 8: Schematic illustration of a proton beam dump experiment on search for $D_{s} \rightarrow \mu N, N \rightarrow \mu \pi$ decay chain: charm mesons $D_{s}$ generated by the proton beam in the target $(T)$ produce a flux of high energy $N^{\prime}$ s through the $U_{\mu N}$ mixing in the decay $D_{s} \rightarrow \mu v_{\mu}$, which penetrate the downstream shielding and decay into $\mu \pi$ pair in a neutrino decay volume (DV). The same setup can be used to search for the process $N \rightarrow \mu e v$. See text.

production cross section $\sigma_{c}$ was measured by LEBC/EHS collaboration revealing $\sigma_{c} \approx 30 \mu \mathrm{b}$ [28]. Thus for $N_{\text {pot }}=10^{20}$ protons incident on the target per year one expects

$$
\frac{\sigma_{c}}{\sigma_{p}} N_{\text {pot }} \sim 0.75 \times 10^{17}
$$

charmed hadrons ( $\sigma_{p} \approx 40 \mathrm{mb}[29]$ is the total proton cross section at SPS energy). With charm branching ratio to sterile neutrino given above (3.1) one expects about

$$
N_{N} \sim 0.75 \times\left(10^{5}-10^{9}\right)
$$

sterile neutrinos crossing the detector each year of operating.

Then, for reference sterile neutrino mass of $1 \mathrm{GeV}$ one estimates the decay length of $10^{2}-10^{5} \mathrm{~km}$ (see Figure 5) and hence

$$
N_{N}^{\mathrm{dec}} \sim 1-10^{7}
$$

decay events per year for $1 \mathrm{~km}$-length detector. Note, that with $P_{T} / P_{L} \sim 1 \mathrm{GeV} / 40 \mathrm{GeV}$ [28] and heavy neutrino following the parent meson travel direction, the detector at a distance of $1 \mathrm{~km}$ has to cover the area of about $25 \mathrm{~m} \times 25 \mathrm{~m}$ to accomplish the task.

Considering a more realistic setup, where detector is placed on the Earth surface and its height is of about $5 \mathrm{~m}$ (cf. the size of CHARM detector [10]) one concludes that the detector has to be about 5 times longer to achieve the same sensitivity to the sterile neutrinos as in the previous conical configuration.

To register all the expected sterile neutrino decays it seems appropriate to install many detectors of a reasonable $5 \mathrm{~m} \times 5 \mathrm{~m} \times 100 \mathrm{~m}$ size rather than one large detector. These small detectors may operate separately by independent groups of experimentalists but together cover the same volume as the large detector, see Figure 8. 


\section{Preliminary Study for the Feasibility of the Proposed Experiment}

The searches for Majorana leptons were undertaken in the past at PS191, BEBC, CHARM, and $\mathrm{NuTeV}$ and led to a negative result, not surprising in view of the cosmological constraints on the properties of sterile neutrinos, that were derived later on. The decays of the vMSM sterile neutrinos are very rare events, and their observation presents a challenge for the detector design and performance. The sensitivity of the search has $\left|U_{\mu N}\right|^{4}$ dependence on the mixing stength, and in order to increase it substantially as compared to the previous searches, one has to use,

(i) higher intensity of the proton beam and larger amounts of pot's,

(ii) shorter distance to the proton target,

(iii) larger $N$ decay fiducial volume,

(iv) longer time of data accumulation,

(v) better efficiency of events detection and reconstruction, background rejection, and so forth.

To compromise the cost of the detector and its performance, we focus mainly on discussions of the experimental setup to search for the $N$ decays in a detector volume filled with a (diluted) gas, not in vacuum. The setup with vacuum requirements would provide possibly better sensitivity, but is more complicated and costly.

The main components of the experimental setup to search for the $N$ decays of sterile neutrino are schematically illustrated in Figure 8. The full detector is composed of single identical modules. Each module consists of a decay volume tank (DV) with diameter of about 5-7 meters and length of 20-30 meters. To minimize cost and amount of passive material, the decay tank could be, for example, a rubber balloon filled with helium. Each DV is followed by a high precision tracker, which could be a layers of straw tube (ST), see, for example, [30]. The entire detector is assembled from $\simeq 50-100$ such modules and is followed by a calorimeter (E/HCAL) which has the electromagnetic and hadronic parts. The detector is protected against charged secondaries from neutrino interactions in the dump by a Veto (V).

The design of a single module is similar to that one used in the PS191, or CHARM experiments, that searched for decays $N \rightarrow v e^{+} e^{-}, \mu \pi, \mu e v, \ldots$ of heavy neutrinos in the $N$ mass range from $10 \mathrm{MeV}$ to $1.8 \mathrm{GeV}$ originated from decays $\pi, K$, and charmed $D$ mesons decays [10-12]. The later experiment, specifically designed to search for neutrino decays in a high-energy neutrino beam, was performed by using $400 \mathrm{GeV}$ protons from the CERN Super Proton Synchrotron (SPS) with the total number of $2.4 \times 10^{18}$ protons on $(\mathrm{Cu})$ target (pot). The CHARM decay detector, located at the distance of $480 \mathrm{~m}$ from the target, consists of decay volume of $3 \times 3 \times 35 \mathrm{~m}^{3}$, three chambers modules located inside the volume to detect charged tracks and followed by a calorimeter. The decay volume was essentially an empty region to substantially reduced the number of ordinary neutrino interactions. The signature of the heavy neutrino decay $v_{h} \rightarrow v e^{+} e^{-}$were events originating in the decay region at a small angle with respect to the neutrino beam axis with one or two separate electromagnetic showers in the calorimeter [10]. No such events were observed and limits were established on the $v_{e, \mu}-v_{h}$ mixing strength as a function of the $v_{h}$ mass. 


\subsection{Production and Decay of Sterile Neutrinos}

If the decays $D_{s}, D \rightarrow \mu N$ exist, one expects a flux of high energy N's from the SPS target, since neutral mesons $D_{s}$ and $D$ are abundantly produced in the forward direction by high energy protons in the target. If $N$ is a relatively long-lived particle, this flux would penetrate the downstream shielding without significant attenuation and would be observed in the proposed detector via the $N \rightarrow \mu \pi, \mu e v$ decay into a high energy $\mu \pi$ or $\mu e$ pair, as schematically illustrated in Figure 7. The experimental signature of the sterile neutrino decays is an observation of either $\mu \pi$ or $\mu$ p pairs originated from a common vertex located in empty space. This signature is clean and the signal events are expected to be selected in the detector with a small background. The occurrence of $N \rightarrow \mu \pi, \mu e v$ decays would appear as an excess of $\mu \pi$ or $\mu e$ pairs in the detector above those expected from standard neutrino interactions. Up to a $N$ mass of $\lesssim 2 \mathrm{GeV}$, the mass difference between the $D_{s}$ and the $\mu, N^{\prime}$ s can originate both from the $v_{\mu}$ produced directly in the $D_{s}$ decay and from the $v_{\mu}$ produced indirectly in the subsequent decay of $\tau$ produced in $D_{s}$ decays. For a $N$ mass below than $\lesssim 1.8 \mathrm{GeV} v_{\mu}$ 's produced in $\tau$ decay can contribute. However these indirect $N^{\prime}$ s have a lower acceptance resulting in a smaller probability to observe the $N$ decays in the detector. Therefore we focus mainly on directly produced $N^{\prime}$ s.

As discussed previously, the mixing between the sterile neutrino and the muon neutrino results in the decay $D_{s} \rightarrow \mu N$, as illustrated in Figure 8. The $D_{s}$ meson, which is normally decays into a $\mu$ and $v_{\mu}$, might instead decay to a $\mu$ and a heavy neutrino $N$. For the mass interval $m_{v_{h}} \lesssim 2 \mathrm{GeV}$ the chirality-flip is mostly due to sterile neutrino mass which results in

$$
\Gamma\left(D_{s} \longrightarrow \mu N\right) \approx \Gamma\left(D_{s} \longrightarrow \mu_{\mu}^{v}\right)\left|U_{\mu N}\right|^{2}\left(\frac{m_{N}}{m_{\mu}}\right)^{2}
$$

In the SM, $D_{s}$ meson decays leptonically via annihilation of the $c$ and $\bar{s}$ quarks through a virtual $W^{+}$. The decay rate of this process is given by

$$
\Gamma\left(D_{s} \longrightarrow l v\right)=\frac{G_{F}^{2}}{8 \pi} f_{D_{s}}^{2} m_{l}^{2} M_{D_{s}}\left(1-\frac{m_{l}^{2}}{M_{D_{s}}^{2}}\right)^{2}\left|V_{c s}\right|^{2},
$$

where the $M_{D_{s}}$ is the $D_{s}$ meson mass, $m_{l}$ is the mass of the charged lepton, $f_{D_{s}}^{2}$ is the decay constant, $G_{F}$ is the Fermi constant, and $V_{c s}$ is a Cabibbo-Kobayashi-Maskawa matrix element which value equals 0.97334 [31]. The decay rate (4.2) is suppressed by the lepton mass squared, since the very leptonic decay is due to chirality-flip. As follows from (4.1), in neutrino scattering experiments the $N$ decay signal rate is proportional to $\alpha\left|U_{\mu N}\right|^{4}$ (the mixing $\left|U_{\mu h}\right|^{2}$ appears twice, through the heavy neutrino production, and through the its decays in the detector) and, thus is more suppressed. The branching ratio of a particular decay mode, for example, $N \rightarrow \mu \pi$ (or $N \rightarrow \mu e v$ ) is given by

$$
\operatorname{Br}(N \longrightarrow \mu \pi)=\frac{\Gamma(\mu \pi)}{\Gamma_{\text {tot }}}=F\left(M_{N},\left|U_{\mu N}\right|^{2}\right),
$$


where the function $F\left(M_{N},\left|U_{\mu N}\right|^{2}\right)$ is calculated in [22], and the total rate $\Gamma_{\text {tot }}$ is dominated by the $N \rightarrow 3 v$ decay channel.

To make quantitative estimates, we performed simplified simulations of the $N$ production in the inclusive reaction

$$
p+B e \longrightarrow D_{s}+X, \quad D_{s} \longrightarrow \mu+N \rightarrow \mu \pi, \mu e v
$$

with the emission of a $\mathrm{GeV}$-scale sterile neutrino $N$ subsequently decaying semileptonically or leptonically in the detector, as illustrated in Figures 7 and 8. In the simulations it is assumed, that the $N$ is a long-lived particle and the flux of $N^{\prime}$ 's penetrates the downstream shielding without significant attenuation. The decay $N \rightarrow \mu \pi, \mu e v$ cannot be distinguished from the antineutrino decay $\bar{N} \rightarrow \mu \pi$ or $\bar{N} \rightarrow \mu e v$ and the obtained result therefore refers to the sum of all these decay modes.

The flux of sterile neutrinos from decays of $D_{s}$ 's and $D^{\prime}$ s produced in the target by primary protons can be expressed as follows:

$$
\Phi(N) \propto N_{\text {pot }} \int \frac{d^{3} \sigma\left(p+N \rightarrow D_{s}(D)+X\right)}{d^{3} p_{D_{s}(D)}} \operatorname{Br}\left(D_{s}(D) \longrightarrow \mu N\right) f d^{3} p_{D_{s}(D)},
$$

where $N_{\text {pot }}$ is the number of pot, $\sigma\left(p+N \rightarrow D_{s}(D)+X\right)$ is the $D_{s}(D)$ meson production cross-section, $\operatorname{Br}\left(D_{s}(D) \rightarrow \mu N\right)$ is the $D_{s}(D) \rightarrow \mu N$ decay mode branching fraction [31], and $f$ is the decay phase space factor, respectively.

To perform quantitative we used simulations of the process shown in Figure 7 by taking into account the relative normalization of the yield of charmed meson species $D_{s}$ and $D$ from the original publications. The invariant cross-section of a charm meson production can be expressed as [31]

$$
E \frac{d^{3} \sigma}{d^{3} p}=\frac{d^{3} \sigma}{p_{T} d p_{T} d y d \phi}=\frac{d^{2} \sigma}{2 \pi p_{T} d p_{T} d y}
$$

where $p_{T}$ is the transverse momentum of the particle, $y$ is its rapidity, and in the last equality integration over the full $2 \pi$ azimuthal angle $\phi$ is performed. For the production cross-sections of $D_{s}$ and $D$ charm mesons we used the Bourquin-Gaillard (B-G) formula from [32]:

$$
E \frac{d^{3} \sigma\left(p+N \rightarrow D_{s}(D)+X\right)}{d^{3} p}=A\left(\frac{2}{E_{T}+2}\right)^{12.3} \exp \left(-\frac{5.13}{Y^{0.38}}\right) f\left(p_{T}\right),
$$

where

$$
f\left(p_{T}\right)= \begin{cases}\exp \left(-p_{T}\right), & p_{T}<1 \mathrm{GeV} \\ \exp \left(-1-\frac{23\left(p_{T}-1\right)}{\sqrt{s}}\right), & p_{T}>1 \mathrm{GeV}\end{cases}
$$

with $Y=y_{\max }-y$, being the rapidity, $s$ is the Mandelstam variable in $\mathrm{GeV}^{2}$, and $E_{T}\left(p_{T}\right)$ is the transverse energy (momentum) in GeV. The coefficient $A$ in (4.7) is a normalization factor 


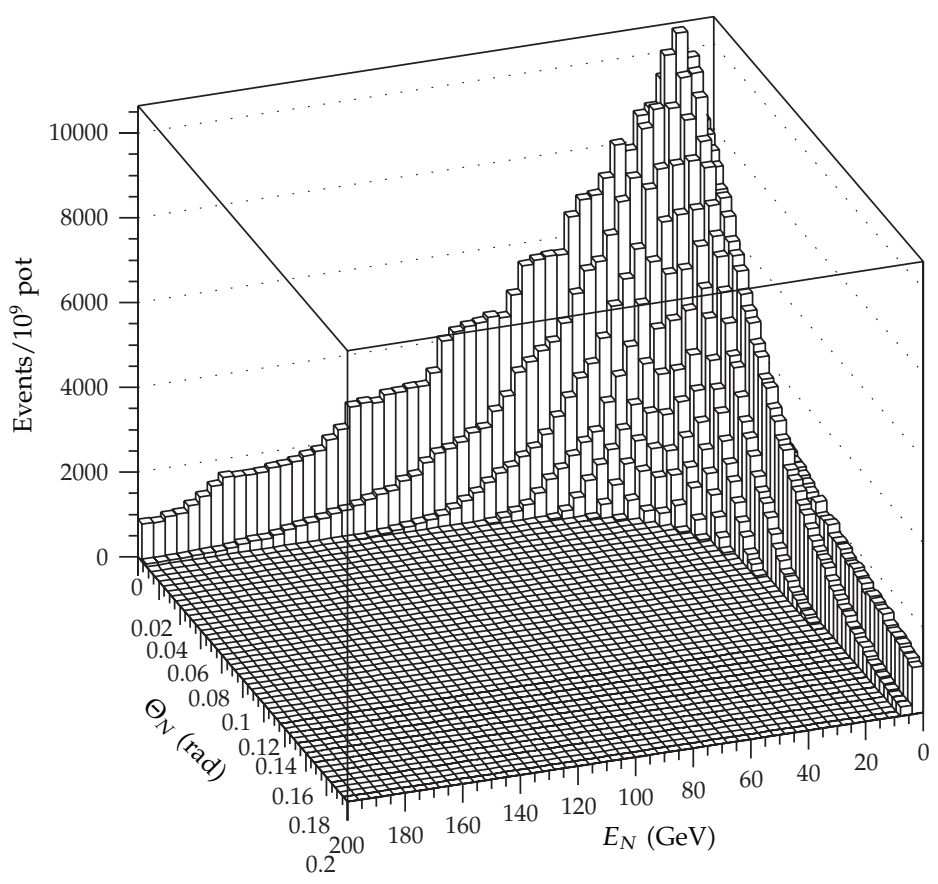

Figure 9: Two dimensional distribution of flux for $1 \mathrm{GeV}$ sterile neutrino as a function of $\Theta_{N}$, the angle between the proton beam axis and momentum of $N$, and its energy calculated for $400 \mathrm{GeV}$ proton incident on Be target. The N's are produced through the decay $D_{s} \rightarrow \mu N$ assuming mixing strength $\left|U_{\mu N}\right|^{2}=1$.

that was tuned to obtain the cross sections of the charm production in pp collisions at $400 \mathrm{GeV}$. The spectra of $\nu_{\tau}$ 's produced in the Be target by primary protons could be calculated by using the approach reported in [33] (see also [34]). However, the Bourquin-Gaillard (B-G) approach gives the parametric form of (4.6) for the production of many different hadrons, including charm, which is in good agreement with data over the full phase-space in highenergy hadronic collisions and with a precision which is satisfactory at the level of our accuracy. The total $D_{s}$ and $D$ production cross sections in $p$-Be collisions were calculated from its linear extrapolation to the target atomic number. The contribution of protons not interacting in the Be target and interacting in the SPS beam dump was not taken into account.

The calculated fluxes and energy distributions of $D_{s}, D$ produced in the Be target were used to predict the flux of $N^{\prime}$ s, as a function of its mass. An example of 2D-flux distribution calculated for $1 \mathrm{GeV}$ sterile neutrino as a function of $\Theta_{N}$, the angle between the proton beam axis and momentum of $N$, and the $N$ energy for $400 \mathrm{GeV}$ proton incident on target is shown in Figure 9. In these calculation the $N^{\prime}$ s are produced through the decay $D_{s} \rightarrow \mu N$ assuming mixing strength $\left|U_{\mu N}\right|^{2}=1$. One can see, that for detector located at the distance $\simeq 100 \mathrm{~m}$ from the target its fiducial area for efficient $N$ detection should be of the order $S \simeq \pi L^{2} \Theta^{2} \simeq 300 \mathrm{~m}^{2}$.

\subsection{Background}

There are several expected sources of background in the experiment, which come from

(i) neutrino interactions in helium,

(ii) neutrino interactions in surrounding materials, 
(iii) cosmics,

(iv) accidentals,

(v) punch-through energetic neutral particles, such as $K_{L}^{0}$ or $n$.

Because of the high number of pot's and many possible sources of background the detail study for the feasibility of the proposed search would require the simulation of a very large number of events resulting in a prohibitively large amount of computer time. Consequently, only small fraction of the required statistics for $v_{\mu} C C(N C)$ inelastic reactions and other background components, for example, such as coherent and quasielastic reactions, and so forth..., were considered for preliminary estimate and combined with numerical calculations.

The estimate shows that the dominant background is expected from the primary $v_{\mu}$ interactions in passive material, which correspond roughly to $\simeq 10^{6} v_{\mu} C C / 10^{20}$ pot events per 1 ton of matter. The largest contribution to background is expected from neutrino interactions yielding a single $\mu \pi$ pair with little hadronic activity in the final state. Neutrino interactions that occur in the surrounding material, for example, upstream of the DV fiducial volume or in material of the decay tanks, also may produce an isolated $\mu \pi$ pairs if the accompanying particles are not detected. These background events from surrounding material could be rejected with an effective method developed in [15], by introducing the collinearity variable $1-\cos \Theta$, where $\Theta$ is the angle between, for example, the $\mu \pi$ pair momentum direction and the neutrino beam axis. The main idea is that if the $\mu \pi$ pair originates from the decay chain $D_{s} \rightarrow \mu N, N \rightarrow \mu \pi$, at high energies the $\mu \pi$ pair momentum extrapolated back should point to the $D_{s}$ production target. An example of the distribution of the variable $1-\cos \Theta$ obtained in the NOMAD experiment, searching for the decay $N \rightarrow e^{+} e^{-} v$, is shown in Figure 5 of [15]. One can see that potentially one could measure the $\mu \pi$ pair momentum direction with precision $1-\cos \Theta \simeq 10^{-5}$. By using this variable, two independent techniques could be used for the background estimation in the signal region. The first is obviously based on the MC. The second method relies on the data themselves. By extrapolating the observed events to the signal region with the shape of the fully simulated MC events a background estimate can be obtained from the data. Using this technique would result in an additional rejection of background events.

\subsection{Sensitivity Estimate}

For a given flux $d \Phi\left(M_{N}, E_{N}, N_{\text {pot }}\right) / d E_{N}$ of $N^{\prime}$ s the expected number of $N \rightarrow \mu \pi, \mu e v$ decays occurring within the fiducial length $L$ of the detector located at a distance $L$ ' from the neutrino target is given by

$$
\begin{aligned}
N_{N \rightarrow \mu \pi, \mu e v}= & \operatorname{Br}\left(D_{s} \longrightarrow \mu N\right) \operatorname{Br}(N \longrightarrow \mu \pi) \int \frac{d \Phi}{d E_{N}} \\
& \cdot \exp \left(-\frac{L^{\prime} M_{N}}{P_{N} \tau_{N}}\right)\left[1-\exp \left(-\frac{L M_{N}}{P_{N} \tau_{N}}\right)\right] \zeta S d E_{N},
\end{aligned}
$$

where $E_{N}, P_{N}$, and $\tau_{N}$ are the $N$ energy, momentum, and the lifetime at rest, respectively, and $\zeta(\simeq 50 \%)$ is the $\mu \pi(\mu e)$ pairs reconstruction efficiency. The acceptance $S$ was calculated by tracing $N^{\prime}$ 's produced in the Be-target to the detector taking the relevant momentum and angular distributions into account. In this estimate the average momentum is $\left\langle p_{N}\right\rangle \simeq 45 \mathrm{GeV}$ and $S \simeq 50 \%$. 


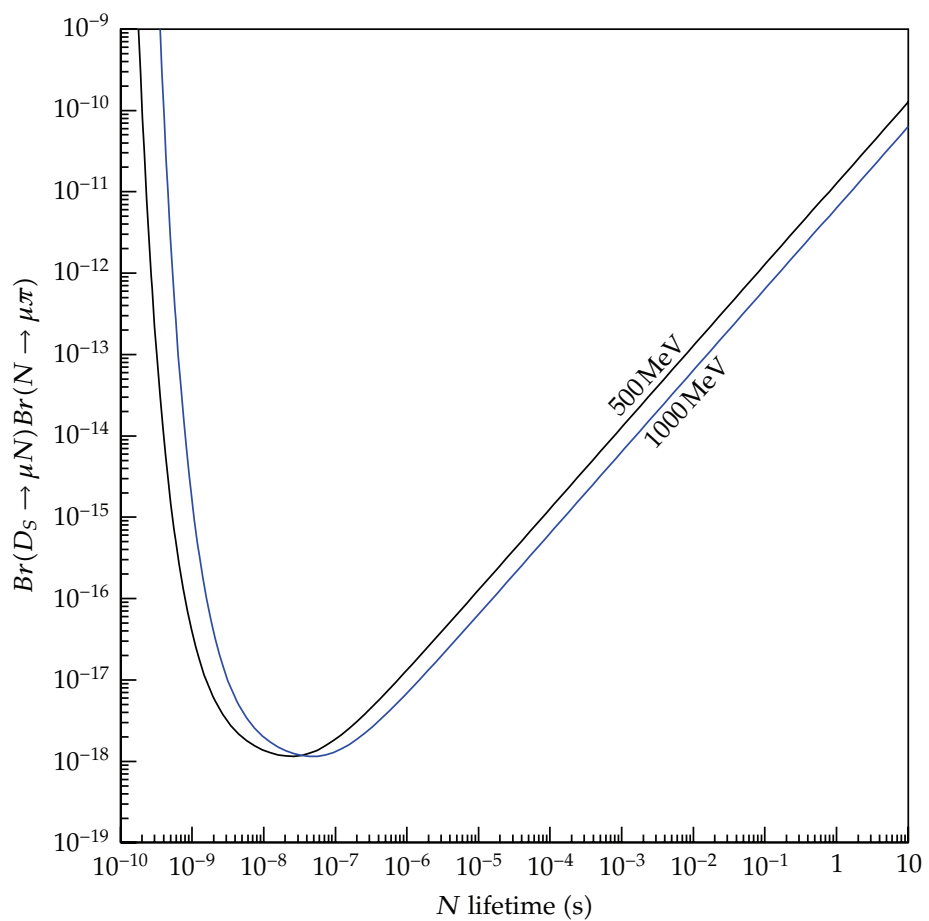

Figure 10: The expected $90 \%$ C.L. upper limits on the branching ratio $\operatorname{Br}\left(D_{s} \rightarrow \mu N\right) \operatorname{Br}(N \rightarrow \mu \pi)$ versus $\tau_{N}$ estimated for the proposed experiment in the background free case. The numbers near the curves indicate the corresponding values of sterile neutrino masses.

In the case of no signal observation, the background free experiment would result in stringent constraints on existence of the sub-GeV sector of the $v$ MSM. An example of the exclusion region calculated in the $\left(\operatorname{Br}\left(D_{s} \rightarrow \mu N\right) \operatorname{Br}(N \rightarrow \mu \pi) \simeq \tau_{N}\right)$ plane for $\simeq 10^{20}$ pot and the detector assembled from $\simeq 10^{2}$ modules, assuming $N_{N \rightarrow \mu \pi, \mu e v}<2.3$ at $90 \% \mathrm{CL}$, is shown in Figure 10. For the mass range $0.5 \lesssim m_{N} \lesssim 2 \mathrm{GeV}$ these limits correspond to the bounds on the mixing strength which are in the range $10^{-6} \lesssim\left|U_{\mu N}\right|^{2} \lesssim 10^{-10}$ and exclude seesaw sterile neutrinos with masses below $2 \mathrm{GeV}$.

Note, that the uncertainty for the $D_{s}$ production at low $p_{T}$ does not significantly affect the sensitivity estimate. For example, the variation of the $D_{s}$ yield in (4.9) by $30 \%$ results in the corresponding variation of the limits on mixing strength $U_{\mu N}$ of order $5 \%$. This is because the sensitivity of the search is proportional to the $\left|U_{\mu N}\right|^{4}$. Indeed, in (4.9) the branching fraction of (4.2) and the decay rate $\Gamma(N \rightarrow \mu \pi, \mu e v)$ of (4.1) both are proportional to $\left|U_{\mu N}\right|^{2}$.

\section{Conclusions}

The energy scale of new physics is not known. If it exists at energies above the Fermi scale (examples include supersymmetry, large or warped extra dimensions, and models with dynamical electroweak symmetry breaking), the search for new particles can be carried out in direct experiments, such as ATLAS and CMS at LHC. In addition, new hypothetical heavy particles inevitably appear as virtual states, leading to different rare processes, absent in the $\mathrm{SM}$. These effects can be found at experiments such as $\mathrm{LHCb}$ and are competitive with the 
direct searches. If no new physics is found at the LHC, this may indicate the absence of new physics all the way between the Fermi and Planck scales.

Quite paradoxically, the largely unexplored up to now domain of energies where the new physics can be hidden is related to physics below the Fermi scale. If the new particles are light and weakly interacting (as in the $v \mathrm{MSM}$, discussed here) then the search for rare processes is superior to high energy experiments. It provides a unique possibility for discovery of new physics, not accessible by any of the LHC experiments.

The present proposal deals with the searches of new relatively light Majorana leptons which "give" the masses to ordinary neutrinos and produce baryon asymmetry of the Universe. We discussed here the parameters of the beam-target experiment which can explore completely the mass range below $2 \mathrm{GeV}$. Due to the weak coupling of these particles to other fermions these experiments are very challenging and require construction of a kilometer scale detector. However, an experiment at much smaller scales, with 100 meters detectors, would be able to explore the dominant fraction of the parameter space.

Below we describe shortly the prospect for other experiments and higher range of sterile neutrino masses.

We start with remark that signal statistics in beam-target experiment proposed in this paper is determined by the fourth power of sterile-active neutrino mixing, $U^{2} \times U^{2}$, since both sterile neutrino production in meson decays and rate of subsequent neutrino decay within a detector are proportional to $U^{2}$. Experiments on searches of meson decays to sterile neutrinos requires (potentially) less statistics of mesons, since the signal statistics are proportional only to the second power of sterile-active neutrino mixing $U^{2}$.

With expected for $v$ MSM branching ratios (3.1) the relevant experiment has to collect not less than $10^{12}$ hadrons in order to fully explore the entire part of parameter space at $M_{N}<2 \mathrm{GeV}$. This statistics is far above what has been obtained at previous $c$ - and $b$-factories $(\mathrm{CLEO}(-c), \mathrm{BaBar}, \mathrm{Belle})$ or at proposed future $c-\tau$ (Novosibirsk) and $B$-factories (Tsukuba, Frascati), where total number of $D$-meson is planned at the level of $10^{9}$, see, for example, [35]. Only at LHC the D-meson production rate is enough to perform this search, however, limits from data taking rate, QCD-background, vertex resolution, particle misidentification etc, prevent measurement of $D$-meson branching ratios at the required level (3.1).

As we have already mentioned, there are no solid-motivated upper limits on sterile neutrino mass of subelectroweak scale. Sterile neutrinos can be heavier than $D$-meson, and then charmed hadrons are out of use. If sterile neutrinos are lighter than $5 \mathrm{GeV}$, they can be produced in beauty hadron decays. This mass range is favorable [27] for a particular mechanism of dark matter production (resonant oscillations in the primordial plasma with lepton asymmetry) in the early Universe. There are other options for dark matter production, which are insensitive to the $1 \mathrm{GeV}$ scale sterile neutrinos, see, for example, [36, 37]. Typical branching ratios of $B$-mesons to sterile neutrino expected within $v$ MSM are [22] by one-two orders of magnitude smaller, than those of $D$-mesons, as both upper and lower bounds on mixing $U^{2}$ decrease with increase of sterile neutrino masses, see Figure 4. Proposed upgrade of LHCb [38] will be able of entering the interesting region of $v$ MSM parameter space by searching for $b$-meson decays into sterile neutrinos, but cannot cover the entire region. Once can further discuss an idea of using LHC-beam ( $4 \mathrm{TeV}, 7 \mathrm{TeV}$, higher) for a beam-target experiment of the kind proposed in this paper, in order to fully explore the $v$ MSM parameter space, if sterile neutrino mass belongs to the the interval $2-5 \mathrm{GeV}$.

The preliminary analysis shows that the quoted sensitivity could be obtained with a proton beam and a detector optimized for several its properties. Namely, (i) the primary beam intensity and the total number of pot's, (ii) the fiducial decay volume of the detector, 
(iii) the composition of the material of decay tank and surrounding material and (iv) the efficiency and precision of the tracking system, (v) the E/HCAL granularity, energy resolution, and particle identification, and (vi) different sources of background a are of importance.

As far as the cost concerned, each module may be relatively cheap, empty-space with simple tracker system either inside or behind the decay volume, and the calorimeter at the far end. Its design may repeat the design of the experiment on precision neutrino measurements and searches for sterile neutrino decays at FNAL [30]. The detector of the 100 length has a sensitivity to $U^{2} \times U^{2}$ worth by a factor only 50 . Thus even one (the first) section will be able to deepen considerably into the parameter range interesting for cosmology, see Figure 4.

\section{Acknowledgments}

The work was supported in part by the Grant of the President of the Russian Federation NS5590.2012 .2 , by the SCOPES program (D.G. and M.S.), and by the RFBR Grant 11-02-01528a (DG).

\section{References}

[1] F. Bezrukov, M. Kalmykov, B. Kniehl, and M. Shaposhnikov, "Higgs boson mass and new physics," In press. http:/ / arxiv.org/abs/1205.2893.

[2] F. Bezrukov, M. Kalmykov, B. Kniehl, and M. Shaposhnikov, " $t-\bar{t}$ factory and Planck physics," Proposal submitted to European Strategy Group, 2012.

[3] M. Shaposhnikov, "Is there a new physics between electroweak and Planck scales?" In press. http:// arxiv.org/abs/0708.3550.

[4] M. Shaposhnikov and D. Zenhäusern, "Scale invariance, unimodular gravity and dark energy," Physics Letters B, vol. 671, no. 1, pp. 187-192, 2009.

[5] M. Shaposhnikov and D. Zenhäusern, "Quantum scale invariance, cosmological constant and hierarchy problem," Physics Letters B, vol. 671, no. 1, pp. 162-166, 2009.

[6] A. Boyarsky, O. Ruchayskiy, and M. Shaposhnikov, "The role of sterile neutrinos in cosmology and astrophysics," Annual Review of Nuclear and Particle Science, vol. 59, p. 191, 2009.

[7] A. Boyarsky, O. Ruchayskiy, and M. Shaposhnikov, "Search for keV-scale sterile neutrinos responsible for dark matter in the universe," Proposal submitted to European Strategy Group, 2012.

[8] T. Yamazaki, T. Ishikawa, Y. Akiba et al., "Search for heavy neutrinos in kaon decay," in Proceedings of the High Energy Physics, vol. 1, Leipzig, Germany, 1984.

[9] A. M. Cooper-Sarkara, S. J. Haywooda, M. A. Parkera et al., "Search for heavy neutrino decays in the BEBC beam dump experiment," Physics Letters B, vol. 160, no. 1-3, pp. 207-211, 1985.

[10] J. V. Allaby, U. Amaldi, G. Barbiellini, C. Berger, F. Bergsma et al., "A search for decays of heavy neutrinos in the mass range 0.5-2.8 GeV," Physics Letters B, vol. 166, no. 4, pp. 473-478, 1986.

[11] G. Bernardi, G. Carugno, J. Chauveau et al., "Search for neutrino decay," Physics Letters B, vol. 166, no. 4, pp. 479-483, 1986.

[12] G. Bernardi, G. Carugno, J. Chauveau et al., "Further limits on heavy neutrino couplings," Physics Letters B, vol. 203, no. 3, pp. 332-334, 1988.

[13] A. Vaitaitis, R. B. Drucker, J. Formaggio et al., "Search for neutral heavy leptons in a high-energy neutrino beam," Physical Review Letters, vol. 83, no. 24, pp. 4943-4946, 1999.

[14] M. Daum, M. Janousch, P.-R. Kettle et al., "KARMEN time anomaly: search for a neutral particle of mass 33.9 MeV in pion decay," Physical Review Letters, vol. 85, no. 9, pp. 1815-1818, 2000.

[15] P. Astier, D. Autiero, A. Baldisseri et al., "Search for heavy neutrinos mixing with tau neutrinos," Physics Letters B, vol. 506, no. 1-2, pp. 27-38, 2001.

[16] P. Achard, O. Adriani, M. Aguilar-Benitez et al., "Search for heavy neutral and charged leptons in $\mathrm{e}^{+}+\mathrm{e}^{-}$annihilation at LEP," Physics Letters B, vol. 517, no. 1-2, pp. 75-85, 2001.

[17] P. Minkowski, " $\mu \rightarrow$ er at a rate of one out of $10^{9}$ muon decays?" Physics Letters B, vol. 67, no. 4, pp. 421-428, 1977.

[18] T. Yanagida, "Horizontal symmetry and masses of neutrinos," Progress of Theorrtical Physics, vol. 64, no. 3, p. 1103, 1980. 
[19] M. Gell-Mann, P. Ramond, and R. Slansky, "Complex spinors and unified theories," in Supergravity, Amsterdam, The Netherlands, 1980.

[20] R. N. Mohapatra and G. Senjanovic, "Neutrino mass and spontaneous parity nonconservation," Physical Review Letters, vol. 44, no. 14, pp. 912-915, 1980.

[21] B. Armbruster, I. Blair, B. A. Bodmann et al., "Anomaly in the time distribution of neutrinos from a pulsed beam stop source," Physics Letters B, vol. 348, no. 1-2, pp. 19-28, 1995.

[22] D. Gorbunov and M. Shaposhnikov, "How to find neutral leptons of the vMSM?" Journal of High Energy Physics, vol. 2007, 2007.

[23] D. Gorbunov and M. Shaposhnikov, "Search for GeV-scale sterile neutrinos responsible for active neutrino masses and baryon asymmetry of the Universe," Proposal submitted to European Strategy Group, 2012.

[24] M. Shaposhnikov, "The vMSM, leptonic asymmetries, and properties of singlet fermions," Journal of High Energy Physics, vol. 2008, 2008.

[25] A. Atre, T. Han, S. Pascoli, and B. Zhang, "The search for heavy Majorana neutrinos," Journal of High Energy Physics, vol. 2009, 2009.

[26] O. Ruchayskiy and A. Ivashko, "Experimental bounds on sterile neutrino mixing angles," Journal of High Energy Physics, vol. 2012, article 100, 2012.

[27] L. Canetti, M. Drewes, and M. Shaposhnikov, "Sterile neutrinos as the origin of dark and baryonic matter," In press. http:/ /arxiv.org/abs/1204.3902.

[28] M. Aguilar-Benitez, W. W. M. Allison, J. L. Bailly et al., "D-Meson production from $400 \mathrm{GeV} / \mathrm{c} \mathrm{pp}$ interactions," Physics Letters B, vol. 189, no. 4, pp. 476-482, 1987.

[29] K. Nakamura, "Review of particle physics," Journal of Physics G, vol. 37, no. 7, Article ID 075021, 2010.

[30] S. R. Mishra, R. Petti, and C. Rosenfeld, "A high resolution neutrino experiment in a magnetic field for project-X at fermilab," High Energy Physics. In press. http:/ / arxiv.org/abs/0812.4527.

[31] J. Beringer, J.-F. Arguin, R. M. Barnett et al., "Review of particle physics," Pysical Review D, vol. 86, no. $1,2012$.

[32] M. Bourquin and J.-M. Gaillard, "A simple phenomenological description of hadron production," Nuclear Physics B, vol. 114, no. 2, pp. 334-364, 1976.

[33] M. C. Gonzalez-Garcia and J. J. Gomez-Cadenas, "Promptv $\tau_{\tau}$ fluxes in present and future $\tau$ neutrino experiments," Physical Review D, vol. 55, no. 3, pp. 1297-1306, 1997.

[34] B. Van de Vyver, "Prompt $V_{T}$ background in wide band $V_{\mu}$ beams," Nuclear Instruments and Methods in Physics Research A, vol. 385, no. 1, pp. 91-99, 1997.

[35] B. Meadows, M. Blanke, A. Stocchi et al., "The impact of SuperB on flavour physics," In press. http:/ / arxiv.org/abs/1109.5028.

[36] M. Shaposhnikov and I. Tkachev, "ThevMSM, inflation, and dark matter," Physics Letters B, vol. 639, no. 5, pp. 414-417, 2006.

[37] F. Bezrukov and D. Gorbunov, “Light inflaton hunter's guide," Journal of High Energy Physics, vol. 2010, 2010.

[38] R. Aaij, C. Abellán Beteta, B. Adeva et al., “Letter of Intent for the LHCb Upgrade,” Tech. Rep. CERNLHCC-2011-001, CERN, Geneva, Switzerland, 2011. 

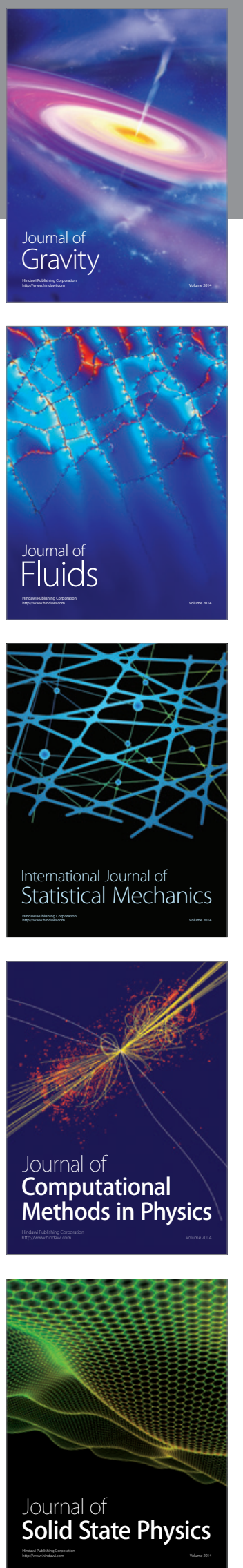

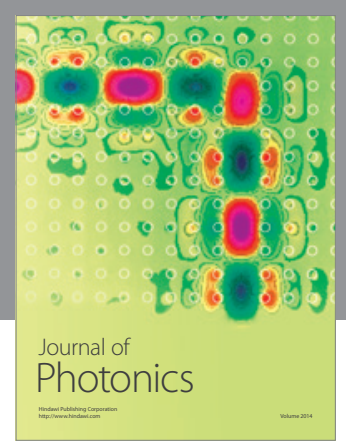

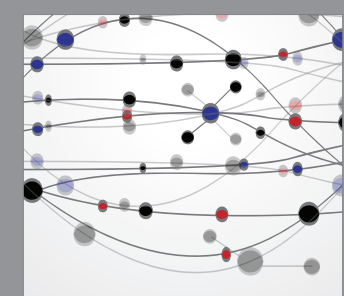

The Scientific World Journal
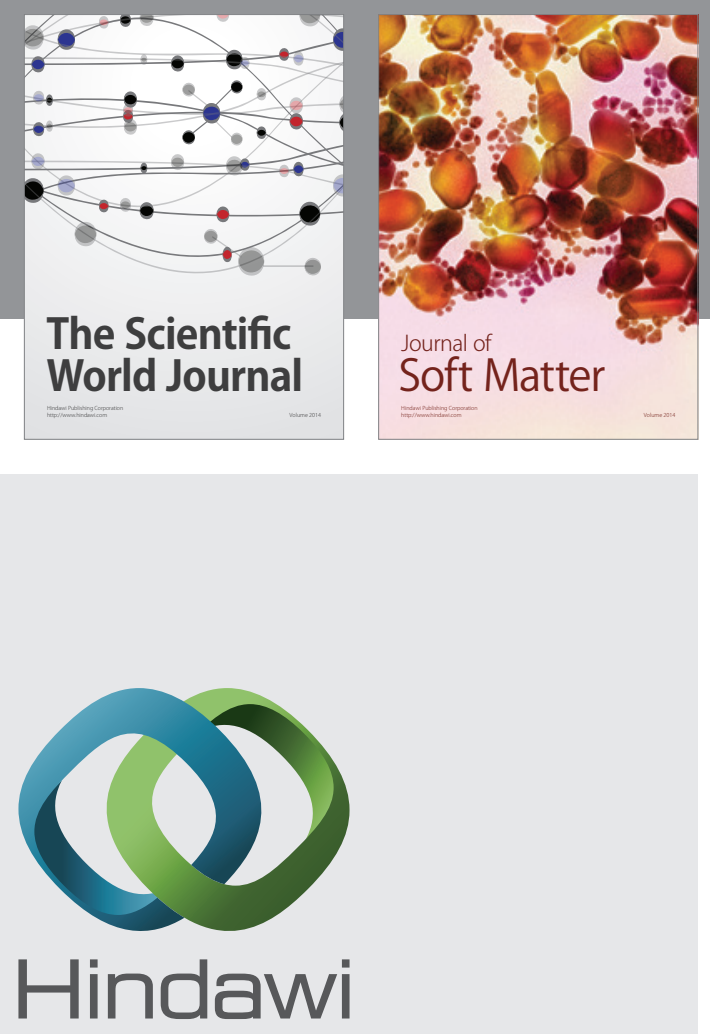

Submit your manuscripts at

http://www.hindawi.com
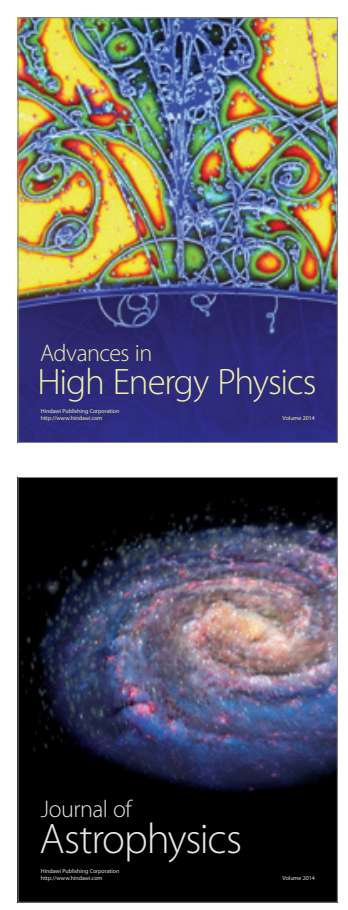
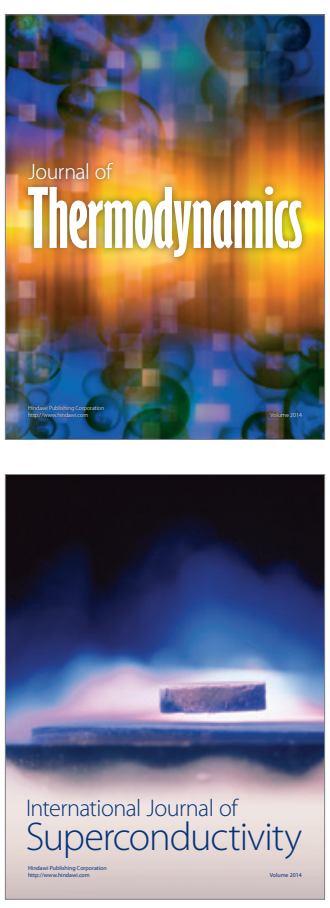
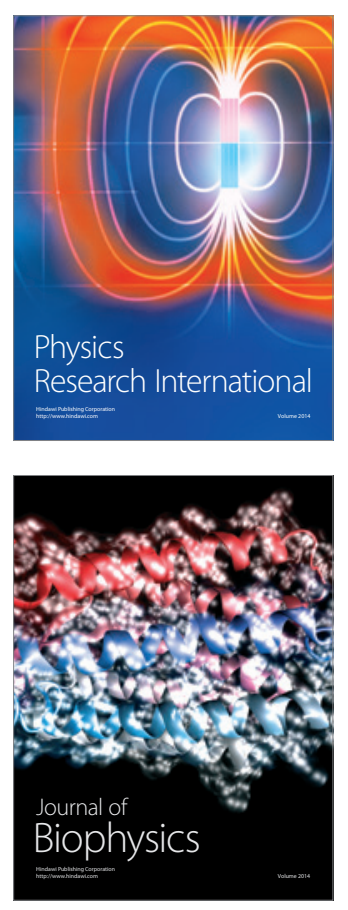
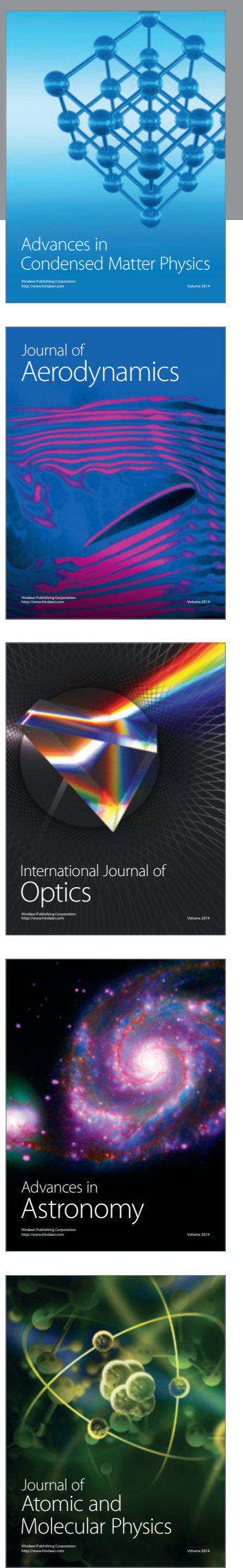